\title{
Incorporación de la plataforma Kahoot en las clases de matemáticas II y evaluación de su funcionamiento
}

\author{
Amanda Carreño ${ }^{1}$, Damián Ginestar $^{2}$ y Esther Sanabria-Codesal ${ }^{2}$ \\ ${ }^{1}$ Instituto de Seguridad Industrial, Radiofísica y Medioambiental. Universitat Politècnica de \\ València \\ ${ }^{2}$ Departamento de Matemática Aplicada. Universitat Politècnica de València
}

\begin{abstract}
This paper presents the experience of introducing the Kahoot tool in the computer practice sessions of the subject Mathematics II of Electronic and Automatic Engineering degree as a motivating element. Furthermore, an analysis of a survey passed to the students of two groups of the subject with different characteristics is made, where different questions related to the tool and its capacity to motivate the students to the revision of the material of the practices before the presential session are included. From the analysis of the obtained results, it can be concluded that, in general, the Kahoot tool is a motivating element for the class, mainly in the part of computer practices.
\end{abstract}

Keywords: Kahoot, gamification, computer practices.

\section{Resumen}

En este trabajo se presenta la experiencia de introducir la herramienta Kahoot en las sesiones de prácticas de informática de la asignatura Matemáticas II de la titulación del grado en Ingeniería Electrónica Industrial y Automática como elemento motivador. Así mismo, se hace un análisis de las opiniones de los alumnos recogidas a través de una encuesta realizada en dos grupos de la asignatura, con distintas características, donde se incluyen preguntas relacionadas con la herramienta y su capacidad de motivar al alumnado en la revisión del material de las prácticas antes de la sesión presencial. Del análisis de los resultados obtenidos se puede concluir que, en general, la herramienta Kahoot es un elemento motivador para la clase, principalmente, en la parte de prácticas informáticas.

Keywords: Kahoot, gamificación, prácticas informáticas. 


\section{Introducción}

La tecnología forma parte de nuestro entorno y desempeña un papel importante en muchos aspectos de nuestra vida cotidiana. Esta rápida evolución e implantación de la nuevas tecnologías en la sociedad actual explica que la mayoría de los estudiantes, que llegan a nuestras aulas, sean nativos digitales acostumbrados a interactuar con ordenadores, programas, teléfonos móviles y apps, durante largos periodos de tiempo.

La aparición de estas nuevas herramientas requieren una adaptación de las metodologías docentes acorde con ellas, que facilite el aprendizaje en este nuevo contexto, por lo que parece indispensable incluir dispositivos digitales en nuestras aulas. Por otro lado, aprovechar las técnicas y estrategias utilizadas por los juegos, desarrollados para este tipo de soportes, puede resultarnos útil para motivar al alumnado con este perfil.

Esta estrategia, conocida como gamificación, se define como "un proceso relacionado con el pensamiento del jugador y las técnicas de juego para atraer a los usuarios y resolver problemas" (Zichermann y Cunningham 2011). Por tanto, la gamificación nos ayudará a involucrar a los alumnos en actividades planteadas desde este enfoque, motivando que estén más activos en el aula y promoviendo así un aprendizaje significativo (Kapp 2012).

En este sentido, proponemos utilizar la herramienta web gratuita Kahoot que permite utilizar la gamificación, a través de los teléfonos móviles de los alumnos en el aula, realizando cuestionarios y obteniendo "feedback" de ellos en tiempo real. Esto resulta muy útil para crear un ambiente dinámico, que fomenta la interacción entre los alumnos, así como entre los alumnos y el profesor en el aula, facilitando la resolución de las dudas o las dificultades que puedan aparecer.

En los últimos años, Kahoot se ha utilizado como elemento dinamizador de la docencia en distintos ámbitos, tanto en la enseñanza secundaria (Pérez Miras 2017; Soto García 2018; Romero Claudio y Álvarez Ramos 2020, etc.), como en la enseñanza superior (Rodríguez-Fernández 2017; Sempere Ferre 2019; Segura y col. 2019, etc.), con resultados positivos. Se concluye así que Kahoot es una herramienta útil para incorporar en el aula, puesto que favorece el apredizaje mejorando el ambiente de trabajo, ya que los alumnos valoran positivamente su parte lúdica.

\section{Objetivos}

Al utilizar docencia inversa en nuestra aulas, uno de los problemas con que nos encontramos más habitualmente es que los alumnos no preparan el material previo, correspondiente a cada sesión presencial. En nuestra asignatura, hemos implementado esta metodología, también conocida como "flip teaching", en las prácticas de laboratorio y proponemos introducir la herramienta Kahoot, en la primera parte de las clases prácticas, con el fin de fomentar la revisión de los contenidos previos a tratar en cada sesión. Con la incorporación de este recurso pretendemos, además de dinamizar el ambiente en el aula, crear una cierta competitividad entre los alumnos que les motive a estudiar los conceptos antes de asistir a la clase. Aunque se trata de una actividad no evaluable, dispone de un sistema de puntos que permite conocer, al final de cada pregunta, la posición del usuario en el ranking global con respecto al resto.

Así, los objetivos concretos que pretendemos alcanzar con esta innovación son los siguientes:

- Introducir la plataforma Kahoot como recurso para el diseño de actividades en el aula. 
- Evaluar el conocimiento adquirido, así como las posibles dificultades de los alumnos en materia, con un formato más atractivo que el ofrecido por la herramienta Exámenes de la plataforma PoliformaT. De esta manera, se añade el factor motivador de la gamificación, lo que genera clases más amenas y una mejor comunicación en las relaciones alumno-alumno y alumnoprofesor.

- Proponer cuestiones básicas (tanto de teoría, como de problemas), para reforzar los conceptos y resolver las posibles dudas, que faciliten una mejor comprensión de los problemas planteados en las prácticas informáticas.

- Repasar comandos o instrucciones del programa Mathematica que ya se deberían conocer y manejar fluidamente al empezar la clase.

- Favorecer el trabajo en equipo.

- Dotar a los alumnos con una herramienta de autoevaluación que los haga conscientes de los aspectos que necesitan reforzar antes de la evaluación.

\subsection{Plataforma Kahoot}

La plataforma Kahoot es una herramienta cada vez más utilizada en las aulas de todos los niveles de enseñanza por su atractivo diseño y fácil uso. Está orientada a realizar cuestionarios con "feedback" en tiempo real. Consta de dos páginas web, una llamada getKahoot, que es la que utiliza el profesor para crear el cuestionario y otra, simplemente Kahoot.it, que es la que los alumnos utilizan para entrar en cada partida a partir de un código numérico, o pin, que le proporciona el profesor.

Kahoot permite crear distintos tipos de preguntas. Aunque la pregunta con 4 posibilidades de respuesta es la más utilizada, también es posible realizar preguntas de verdadero o falso, entre otras posibilidades. La puntuación de las preguntas se puede modificar, entre varias opciones predeterminadas por la aplicación, al igual que ocurre con el tiempo límite para contestarlas. Además se pueden insertar imágenes en las preguntas, tanto predeterminadas, como elaboradas por el profesor, lo que da mucha versatilidad al formato. La Figura 1 muestra la apariencia de una pregunta del juego, tal y como se muestra a los alumnos, en este caso del grupo ARA (que se imparte en inglés).

Existen otras plataformas similares para crear cuestionario, por ejemplo Quizizz, que no presenta límites fijos en el tiempo de respuesta, lo que puede resultar útil para algunas actividades, pero no resulta tan atractiva como Kahoot para los alumnos (Ankit 2015; Göksün y Gürsoy 2019). La plataforma Plickers (Plickers 2019) es un poco diferente a las anteriores, ya que cada estudiante tiene un conjunto de tarjetas con códigos QR que corresponden a las posibles respuestas. Por último, ThatQuiz (ThatQuiz 2019) permite generar actividades repetitivas de reconocimiento, comparación y operatividad en diversos ámbitos de las matemáticas (llamadas exámenes en la aplicación) y es muy utilizada en los niveles más básicos. 


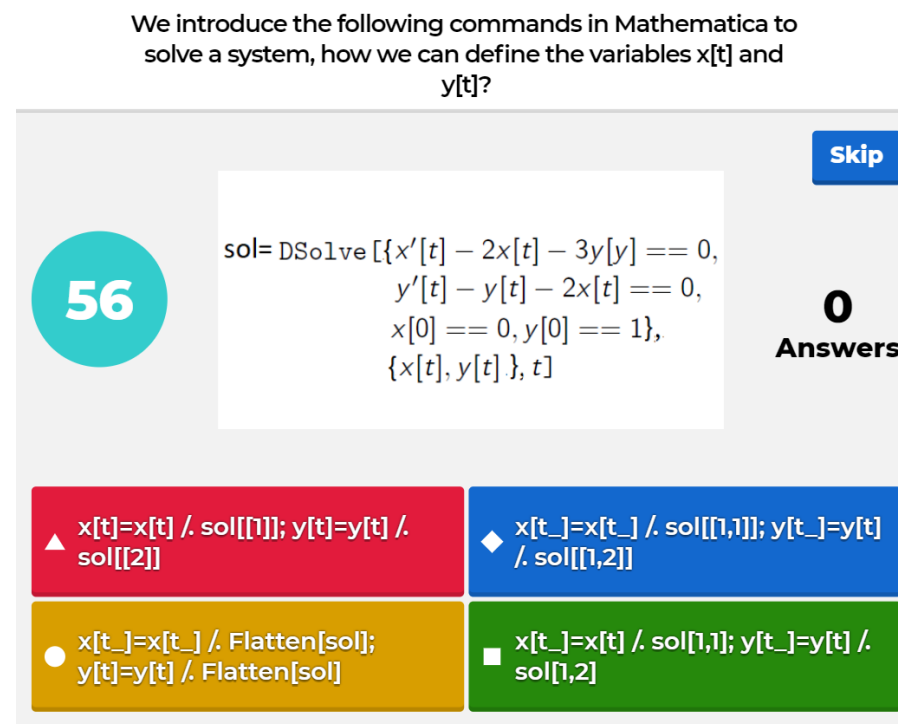

kahoot.it Game PIN: $\mathbf{7 3 2 5 0 3 1}$

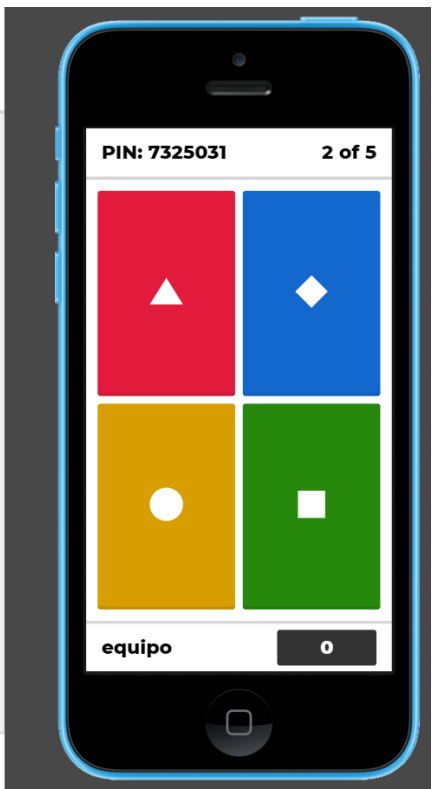

Fig. 1: Vista del alumno de la plataforma Kahoot!.

\section{Desarrollo de la innovación}

La propuesta presentada en este trabajo se ha desarrollado en la asignatura Matemáticas II del Grado en Electrónica Industrial y Automática, impartido en la Escuela Técnica Superior de Ingeniería del Diseño (ETSID) de la Universitat Politècnica de València (UPV).

Matemáticas II es una asignatura obligatoria de 6 créditos ECTS, repartidos en 3 créditos de teoría, 1 de práctica de aula y 2 de prácticas informáticas. Los contenidos que se trabajan en ella son: Series de Fourier, Ecuaciones Diferenciales ordinarias y Transformadas directas e inversas de Laplace.

La incorporación de la plataforma Kahoot se ha realizado en la parte correspondiente a las prácticas informáticas de la asignatura Matemáticas II, donde aplicamos la metodología de docencia inversa. La experiencia se ha valorado en dos grupos de prácticas con perfiles muy diferentes, el grupo 321 de alto rendimiento académico (ARA), en el que la docencia se imparte en inglés y tiene un número reducido de alumnos con buen expediente académico y el grupo 326 de tarde, donde la docencia se imparte en castellano con un mayor número de alumnos, algunos de los cuales trabajan.

Las prácticas se realizan en un laboratorio con ordenadores, donde utilizamos el software Mathematica, un programa intuitivo y versátil, con un uso muy extendido en el ámbito del cálculo simbólico, y que resulta una herramienta útil para la resolución (de forma analítica y numérica) de los ejercicios planteados durante el curso. El programa además dispone de una amplia documentación y mucha ayuda online. Es, por ello, que las prácticas les sirven a los alumnos, tanto para plantear y reforzar los problemas relacionados con la asignatura, como para mejorar el manejo del software.

Durante el semestre en que se imparte la asignatura, realizamos las siguientes 7 prácticas: 
1. Repaso del programa Mathematica.

2. Series de Fourier.

3. Ecuaciones diferenciales de orden 1.

4. Ecuaciones diferenciales de orden $n$.

5. Sistemas de ecuaciones diferenciales.

6. Métodos numéricos para ecuaciones diferenciales.

7. Transformada de Laplace.

Como ya hemos comentado anteriormente, Matemáticas II está incluida en el proyecto institucional de Docencia Inversa de la UPV y nuestra experiencia al respecto está siendo satisfactoria (Coll Aliaga y col. 2017; Coll Aliaga y col. 2016), puesto que con esta metodología conseguimos implicar más al alumnado.

El desarrollo de la actividad en las prácticas se divide en tres fases:

- Trabajo en casa. En la primera fase, los alumnos deben revisar el material disponible en la plataforma PoliformaT, que incluye explicaciones sobre los comandos de Mathematica que vamos a utilizar en la sesión, así como ejemplos y ejercicios propuestos para que el alumno pueda practicar. Este material está en formato "screencast", es decir, capturas de vídeo con voz en off apoyados con documentos en formato texto, o bien únicamente con documentos explicativos, dependiendo del grupo al que pertenezcan.

- Kahoot. La segunda fase se da al inicio de la sesión presencial en el aula informática. Antes de comenzar con el Kahoot se dejan unos minutos para preguntar dudas acerca del material de la práctica que los alumnos han trabajado en casa y reforzar los conceptos que el profesor considere claves para dicha sesión. Tras ello, los alumnos se dividen en grupos de 3 o 4 personas (que mantienen durante todo el curso con el fin de hacer una liga y proclamar al final de curso el equipo ganador) y comienza el cuestionario preparado con Kahoot. El profesor facilita el código para conectarse al Kahoot y, una vez los grupos se han dado de alta, se muestran sucesivamente las preguntas a través de la pantalla del proyector del aula. Los grupos las contestan en su teléfono móvil, a través de un código de colores, sin necesidad de instalar la aplicación. Al final de cada pregunta se muestra la solución correcta y la puntuación obtenida por cada equipo. Esta parte es importante, porque conocer la posición de cada uno en el ranking, es lo que aumenta la competitividad entre los equipos y su motivación para hacerlo bien. Además en este punto, el profesor (o algún alumno voluntario) expone la justificación de la respuesta correcta frente al resto, lo que favorece aclarar cualquier concepto erróneo o duda que pudiera aparecer. Al terminar las preguntas, la plataforma muestra la clasificación general obtenida por los grupos.

- Examen PoliformaT. La última fase corresponde a la evaluación de la práctica. Se lleva a cabo mediante la herramienta Exámenes de la plataforma PoliformaT donde se plantean ejercicios que los alumnos tienen que resolver de manera autónoma e individual.

La incorporación de la herramienta Kahoot permite al alumno autoevaluarse y reforzar conceptos teóricos y prácticos de una manera amena y dinámica. Además motiva al alumno a prepararse la práctica antes de la sesión presencial, ya que al utilizar la plataforma se dispone del tiempo justo 
para dar la respuesta y no es posible leer el material previo de la práctica a la vez que se resuelven las cuestiones planteadas.

\section{Resultados}

En esta sección vamos a analizar la valoración que los alumnos hacen de herramienta Kahoot en las sesiones de prácticas de la asignatura. El análisis se ha llevado a cabo con los datos de una encuesta, realizada al final del cuatrimestre (ver Anexo A para más detalles), que recoge las percepciones de los alumnos sobre el dinamismo y la motivación de la clase tras la inclusión de los cuestionarios realizados con Kahoot, así como sugerencias de mejora para el futuro. Además de las respuestas en la encuesta, hemos tenido en cuenta la nota obtenida por los alumnos en el primer parcial de las prácticas informáticas y el examen de teoría de la asignatura. La valoración se ha realizado en la escala de Likert 1 a 5 (siendo 1. Muy en desacuerdo, 2. Un poco desacuerdo, 3. Ni acuerdo ni desacuerdo, 4. Bastante de acuerdo, 5. Muy de acuerdo).

La encuesta consta de 16 preguntas y el enunciado de cada una de ellas, desde la Q1 a la Q16, está expuesto en la Figura 6. Hemos dividido dicha encuesta en varios bloques: el primero, que abarca desde la pregunta Q1 hasta la pregunta Q8, corresponde a la valoración de la experiencia de introducir los cuestionarios de Kahoot en las prácticas; el segundo bloque recoge las opiniones referidas al trabajo en grupo y está formado por las preguntas Q9 y Q10; el tercer bloque se centra en las principales motivaciones del alumno (preguntas Q11, Q12 y Q13) y, por último, las preguntas Q14, Q15 y Q16 indican la adecuación de utilizar los cuestionarios de la plataforma Kahoot como sistema de evaluación. Las últimas preguntas del cuestionario, fuera de la escala Likert, se refieren a la nota obtenida en el primer parcial de la teoría y prácticas de aula, así como en las prácticas informáticas.

Para la valoración de los resultados se ha pasado la encuesta en dos grupos, con perfil muy diferente, el grupo ARA y el grupo 326. Del primer grupo se han recogido 13 encuestas de un total de 23 alumnos matriculados (56,5\% de participación), mientras que del segundo grupo se han recogido 10 encuestas de un total 30 matriculados (33,3\% del alumnado). La baja participación de los alumnos en las encuestas se debe principalmente a la menor asistencia generalizada (especialmente en el grupo de la tarde) durante los últimos días del cuatrimestre, antes de comenzar lo exámenes, momento en el que se realizaron las encuestas.

Antes de comenzar el análisis mediremos la confiabilidad de la escala utilizada en nuestra encuesta, en la que se han unificado las respuestas de los dos grupos, mediante el test Alfa de Cronbach (Cronbach 1951) ampliamente utilizado con este fin en el análisis de los resultados para este tipo de encuestas. Este valor se suele interpretar como una medida unidimensional de una escala, es decir, si estamos midiendo de manera consistente algo con ella. Para ello, usamos la función alpha del paquete psych del software R (Revelle 2017). El resultado obtenido es 0.8 con un $95 \%$ de confianza. Este dato indica una buena consistencia interna, es decir, los ítems están dirigidos en la misma dirección.

Para una descripción más detallada de los resultados de las encuestas se ha utilizado el paquete Likert de R (Bryer y Speerschneider 2016). Los resultados obtenidos se muestran en la Figura 2. Esta Figura incluye la media y la desviación típica obtenida (como si fuesen variables continuas), así como el porcentaje de resultados obtenido para cada variable. En líneas generales, los alumnos se muestran bastante de acuerdo con las premisas que han tenido que valorar. En particular, la parte donde más de acuerdo están los alumnos es que su motivación, en general, se debe principal- 
mente a aprobar el examen (Motivates_pass_exam), lo que hace que la inclusión del Kahoot como elemento no evaluador en algunos casos no resulte tan efectivo como pretendíamos inicialmente. En cualquier caso, las valoraciones positivas en la parte de motivación para preparar las prácticas (Motivates_prepare_practice) superan las valoraciones negativas.

\begin{tabular}{|c|c|c|c|c|c|c|c|}
\hline Q2.Useful_review_theory & $3.91(0.79)$ & $0.0 \%$ & $8.7 \%$ & $8.7 \%$ & $65.2 \%$ & $17.4 \%$ & \multirow{6}{*}{ Percent } \\
\hline Q3.Useful_review_Mathematica & $3.91(0.73)$ & $0.0 \%$ & $4.3 \%$ & $17.4 \%$ & $60.9 \%$ & $17.4 \%$ & \\
\hline Q14.Evaluate_in_theory & $3.74(1.25)$ & $4.3 \%$ & $17.4 \%$ & $13.0 \%$ & $30.4 \%$ & $34.8 \%$ & \\
\hline Q8.Apply_in_other_subjects & $4.09(0.95)$ & $0.0 \%$ & $8.7 \%$ & $13.0 \%$ & $39.1 \%$ & $39.1 \%$ & \\
\hline Q11.Motivates_podium & $4.04(1.11)$ & $0.0 \%$ & $17.4 \%$ & $4.3 \%$ & $34.8 \%$ & $43.5 \%$ & \\
\hline Q13.Motivates_pass_exam & $4.70(0.76)$ & $0.0 \%$ & $4.3 \%$ & $4.3 \%$ & $8.7 \%$ & $82.6 \%$ & \\
\hline Q4.Motivates_prepare_practice & $3.30(1.02)$ & $4.3 \%$ & $13.0 \%$ & $43.5 \%$ & $26.1 \%$ & $13.0 \%$ & \\
\hline Q7.Kahoot_easier_than_exam & $3.78(0.85)$ & $0.0 \%$ & $4.3 \%$ & $34.8 \%$ & $39.1 \%$ & $21.7 \%$ & \\
\hline Q10.Help_groupmates & $4.39(0.78)$ & $0.0 \%$ & $0.0 \%$ & $17.4 \%$ & $26.1 \%$ & $56.5 \%$ & 50 \\
\hline Q9.Work_group_better & $4.43(0.73)$ & $0.0 \%$ & $0.0 \%$ & $13.0 \%$ & $30.4 \%$ & $56.5 \%$ & 25 \\
\hline Q12.Motivates_good_marks & $4.35(0.93)$ & $0.0 \%$ & $8.7 \%$ & $4.3 \%$ & $30.4 \%$ & $56.5 \%$ & \\
\hline Q1.Funny & $4.39(0.66)$ & $0.0 \%$ & $0.0 \%$ & $8.7 \%$ & $43.5 \%$ & $47.8 \%$ & 0 \\
\hline Q6.Classes_more_dynamical & $4.74(0.45)$ & $0.0 \%$ & $0.0 \%$ & $0.0 \%$ & $26.1 \%$ & $73.9 \%$ & \\
\hline Q16.Evaluate_in_CPU_practices & $4.30(0.82)$ & $0.0 \%$ & $4.3 \%$ & $8.7 \%$ & $39.1 \%$ & $47.8 \%$ & \\
\hline Q15.Evaluate_in_classroom_practices & $3.87(1.10)$ & $0.0 \%$ & $17.4 \%$ & $13.0 \%$ & $34.8 \%$ & $34.8 \%$ & \\
\hline Q5.Help_to_autoevaluate & $3.74(0.86)$ & $0.0 \%$ & $4.3 \%$ & $39.1 \%$ & $34.8 \%$ & $21.7 \%$ & \\
\hline & Mean (SD) & 1 & 2 & 3 & 4 & 5 & \\
\hline
\end{tabular}

Fig. 2: Porcentaje obtenido para cada respuesta, junto con la media (Mean) y la desviación típica (SD), de todas las variables de la encuesta.

Para describir con más detalle las variables relacionadas con la experiencia con la herramienta Kahoot, la Figura 3 muestra los diagramas de barras para cada variable. La zona verde indica los resultados positivos (puntuación 4 o 5), mientras que la zona amarilla los negativos (puntuación 1 o 2). En ella se puede observar una valoración positiva en todas las variables que se han consultado, destacando principalmente la capacidad del cuestionario Kahoot para hacer las clases más dinámicas (Classes_more_dynamical) y divertidas (Funny) y su utilidad para repasar conceptos, tanto de la teoría, como de las prácticas de la asignatura (Useful_review_theory, (Useful_review_Mathematica)).

Uno de los aspectos que más nos preocupan como docentes es la motivación de los alumnos. Por ello, hemos considerado hacer un análisis especial para comprobar cuál es la principal causa por la que lo alumnos intentan obtener mejores resultados en el Kahoot. La Figura 4 muestra las funciones de densidad para las variables, "estar en el podium"(Motivates_podium), "obtener buenas notas"(Motivates_good_marks) y "aprobar el examen"(Motivates_pass_exam). En ella, se observa que la razón que más pesa en el alumnado es la de aprobar el examen.

A la vista de los resultados de la Figura 2, la introducción del Kahoot en las prácticas mejora la motivación para preparar las prácticas antes de la clase presencial. Sin embargo, existe un $13 \%$ de alumnos que no están muy de acuerdo con esta afirmación. Veamos, a continuación, si esta valoración tiene alguna relación con las notas obtenidas en la asignatura. Como el número de muestras es relativamente pequeño, propondremos un test no paramétrico para su análisis. En particular, utilizaremos un test de muestras emparejadas, ya que utilizaremos dos muestras de un mismo individuo para comparar estas dos variables. A este test se le conoce como Test de los signos o de Wilcoxon (Cuzick 1985). Primero realizamos un test para comprobar si existe alguna relación entre la motivación para la preparación de las prácticas (Motivates_prepare_practice) y la nota obtenida en teoría y prácticas de aula. Para ello, proponemos un test cuya hipótesis nula es que ambas variables tienen la misma distribución y como hipótesis alternativa que no la tienen. 


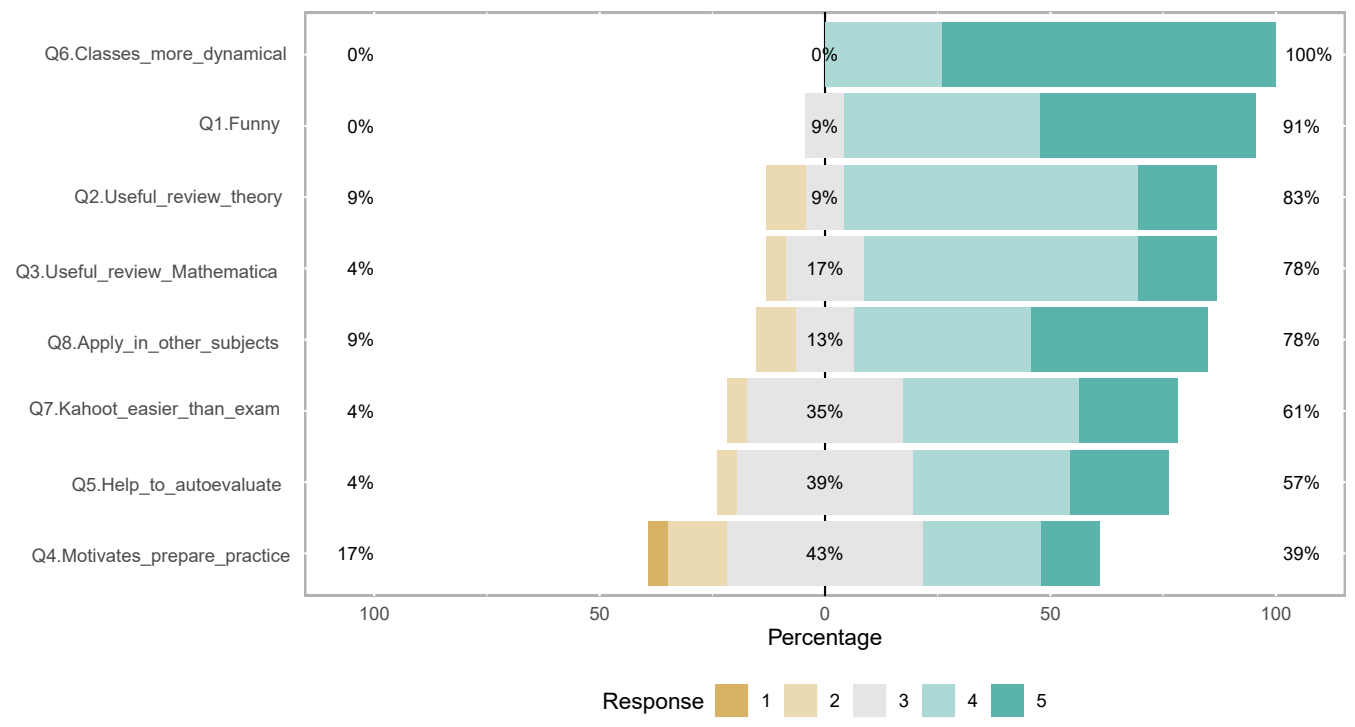

Fig. 3: Diagrama de barras para la valoración del Kahoot como experiencia en las aulas.

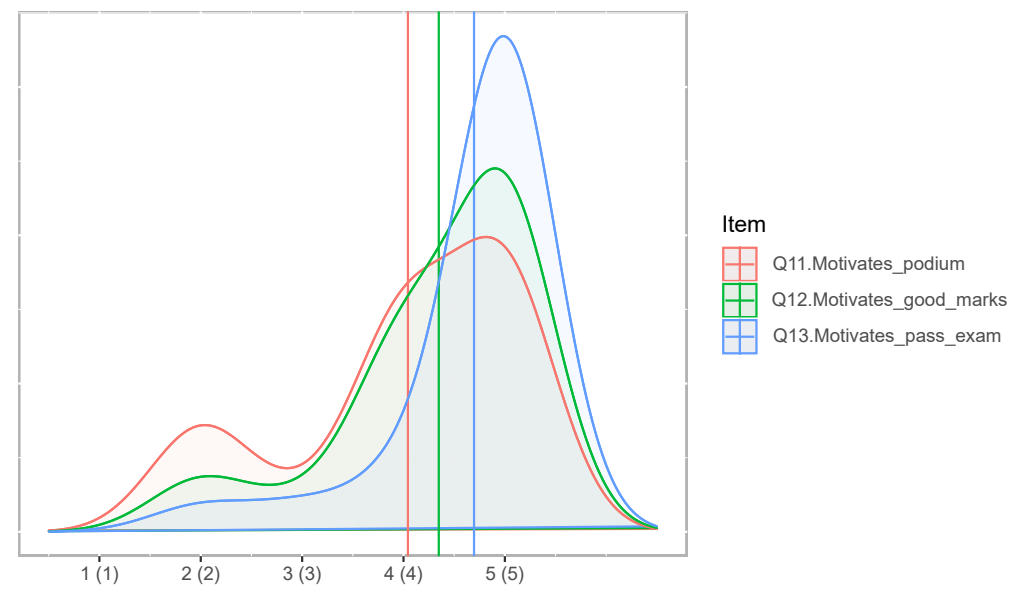

Fig. 4: Curvas de densidad de probabilidad sobre los aspectos relacionados con la motivación.

Este test se puede escribir como

$$
\left\{\begin{array}{l}
H_{0}: \text { mediana }_{y_{\text {mark_theory }}}=0, \\
H_{A}: \text { mediana }_{y_{\text {mark_theory }}} \neq 0,
\end{array}\right.
$$

donde $y_{\text {mark_theory }}$ es la variable diferencia $y_{\text {mark_theory }}=$ motivates-mark.theory. En general, los tests no paramétricos se plantean en términos de las medianas. 
El p-valor obtenido es $0,982 \mathrm{y}$, por tanto, no existe evidencia estadística para afirmar que hay diferencias entre ambas distribuciones. Es decir, sí que hay una relación entre las notas obtenidas en teoría y práctica con la valoración del Kahoot para motivar la preparación de las clases.

De igual forma, analizamos la relación entre la motivación para la preparación de la práctica (Motivates_prepare_practice) con la nota obtenida en las prácticas informáticas. Igual que en el caso anterior, planteamos un test de Wilcoxon cuya hipótesis nula es afirmar que ambas variables tienen la misma distribución y la hipótesis alternativa lo contrario. Este test se puede escribir como

$$
\left\{\begin{array}{l}
H_{0}: \text { mediana }_{y_{\text {mark_pract }}}=0, \\
H_{A}: \text { mediana }_{y_{\text {mark_pract }}} \neq 0,
\end{array}\right.
$$

donde $y_{\text {mark_pract }}$ es la variable diferencia $y_{\text {mark_pract }}=$ motivates-qualification.pract

En este caso el p-valor obtenido es 0,008449 y, por tanto, sí existe evidencia estadística para rechazar la hipótesis nula y deducimos que no hay diferencias entre ambas distribuciones. En este caso, concluimos que no hay ninguna relación entre la valoración sobre la motivación para preparar las prácticas informáticas con la nota obtenida en éstas.

De cara a incorporar, como posibilidad futura; los cuestionarios del Kahoot como parte evaluadora de la asignatura, se les ha preguntado a los alumnos su opinión sobre el interés de su introducción en las distintas partes de la asignatura y así, adecuar los contenidos del Kahoot! para profundizar en conceptos relacionados con teoría, práctica de aula (ejercicios prácticos resueltos a mano) y prácticas informáticas (ejercicios prácticos resueltos mediante el software Mathematica). Las opciones estarían entre teoría (Evaluate_in_theory), prácticas de aula (Evaluate_in_classroom_practicas) o prácticas informáticas (Evaluate_in_CPU_practicas). En la Figura 5 están representados los diagramas de barras obtenidos para las variables anteriores. En ella, se puede ver una clara tendencia positiva de los alumnos a que el Kahoot sea un elemento evaluador, sobre todo en las prácticas informáticas.

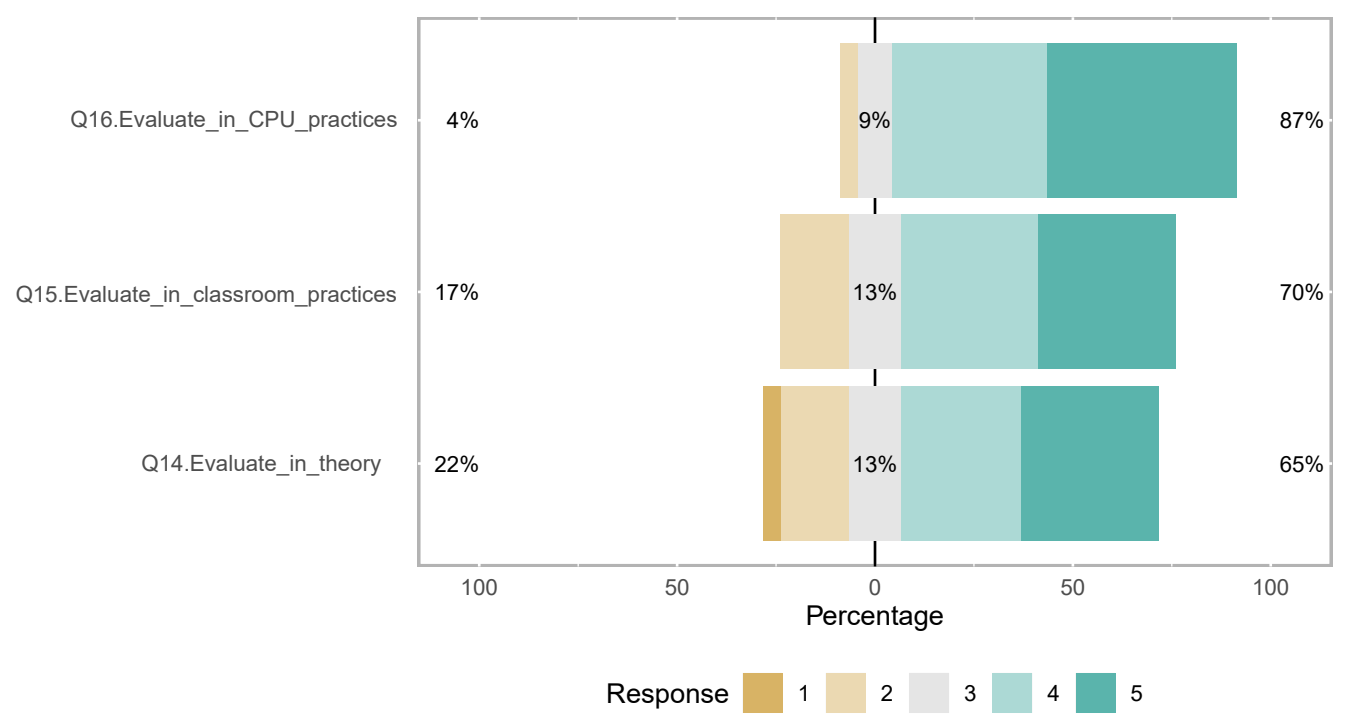

Fig. 5: Diagrama de barras para la valoración del Kahoot como sistema evaluador en futuros cursos académicos. 
Por último, estudiaremos si existen diferencias entre los dos grupos analizados para las variables de experiencia y trabajo en grupo. Como antes, al disponer de pocos datos utilizaremos un test no paramétrico. Sin embargo, en este caso, para cada variable se ha tomado la muestra a distintos individuos y, por tanto, se trata de muestas no emparejadas. Plantearemos pues diferentes test de Mann-Whitney (McKnight y Najab 2010), donde consideramos como hipótesis nula que la variable analizada tiene una misma distribución para los dos grupos o la hipótesis alternativa que ambas tienen una distribución distinta. Este test se puede expresar de la siguiente forma

$$
\left\{\begin{array}{l}
H_{0}: \text { mediana }_{\mathrm{ARA}}=\text { mediana }_{326}, \\
H_{A}: \text { mediana }_{\mathrm{ARA}}=\text { mediana }_{326},
\end{array}\right.
$$

donde las medianas a comparar serán las distintas variables relacionadas del bloque relacionado con la experiencia y con el trabajo en grupo. La Tabla 1 recoge los valores de la mediana para ambos grupos y el p-valor obtenido en cada uno de los contrastes. Los resultados obtenidos muestran que no existen diferencias entre los dos grupos salvo para dos variables con una confianza del $95 \%$. La primera es que el grupo ARA no considera de la misma manera que el grupo 326 que las preguntas de Kahoot sean más fáciles que las preguntas del examen que luego se usa para la evaluación. A la vista de la mediana se puede observar como el grupo 326 considera más sencillas las preguntas del Kahoot que las del examen, mientras que el grupo ARA no aprecia tanta diferencia. La siguiente diferencia se observa en el trabajo en grupo. Ambos grupos no piensan de la misma manera en cuanto al beneficio del trabajo en grupo, que permite ayudarse unos a otros. De hecho, si observamos la mediana de las variables relacionadas con el trabajo en grupo, observamos que al grupo ARA le gusta trabajar en grupo y ayudarse entre ellos mientras que el grupo 326 no está tan de acuerdo con esta organización (aunque también se muestra positivo en líneas generales).

Tabla 1: Comparación de las variables para el grupo ARA y el grupo 326.

\begin{tabular}{lrrr}
\hline Variable & Mediana ARA $_{\text {Aediana }}$ & M26 & p-value \\
\hline Q1. Funny & 5 & 4 & 0.18 \\
Q2. Useful_review_theory & 4 & 4 & 0.49 \\
Q3. Useful_review_Mathematica & 4 & 4 & 0.29 \\
Q4. Motivates_prepare_practice & 3 & 3 & 0.39 \\
Q5. Help_to_autoevaluate & 4 & 4 & 0.47 \\
Q6. Classes_more_dynamical & 5 & 5 & 0.41 \\
Q7. Kahoot_easier_than_exam & 3 & 4 & 0.03 \\
Q8. Apply_in_other_subjects & 4 & 4 & 0.34 \\
Q9. Work_group_better & 5 & 4 & 0.29 \\
Q10. Help_groupmates & 5 & 4 & 0.03 \\
\hline
\end{tabular}

\section{Conclusiones}

Una vez analizadas las opiniones de los alumnos, sobre la incorporación de los cuestionarios de Kahoot, para mejorar la implantación de la docencia inversa en las prácticas informáticas de la asignatura Matemáticas II, podemos concluir que la mayoría de nuestros objetivos iniciales se han cumplido, ya que en general el planteamiento de esta actividad en el aula: 
- Ha tenido una buena valoración entre los alumnos a la hora de dinamizar la docencia, de manera que la gamificación ha resultado un factor motivador para trabajar los conceptos de la asignatura, así como para resolver dudas y repasar conceptos, aunque no ha tenido tan buenos resultados como esperábamos a la hora de preparar las sesiones prácticas.

- Ha favorecido el trabajo en equipo y les ha animado, a través de la autoevaluación a mejorar sus resultados, ya que les ayuda en su objetivo principal de aprobar el examen para superar la asignatura.

Estos resultados coinciden con la mayoría de análisis realizados por otros autores sobre esta herramienta, por lo que consideraremos extenderla también al ámbito de las clases teóricas en sucesivos cursos académicos.

Por otro lado, como elementos a mejorar, nos parece interesante aumentar el nivel de las preguntas para que sean lo más parecidas posible a las del examen para, de esta forma, animarlos a prepararse mejor las sesiones previas y así aprovechar más las sesiones de prácticas en el aula y mejorar así el aprendizaje de la asignatura. 


\section{A Anexo}

La Figura 6 muestra la encuesta que han realizado los alumnos para el análisis de la incorporación de la plataforma Kahoot en las clases de Matematicas II.

\section{Kahoot Survey}

I agree. . .

As experience...
Q1. It is funny $\ldots \ldots \ldots \ldots \ldots \ldots \ldots \ldots \ldots \ldots \ldots \ldots \ldots$
Q2. It is useful to review the theory concepts .......
Q3. It is useful to review the Mathematica commands
Q4. It motivates to prepare the practices at home ....
Q5. It is good to auto-evaluate your work ..........
Q6. It makes the classes more dynamical ............
Q7. Kahoot questions are easier than the exam exercises
Q8. I would like to use it also in other subjects ........

\section{Working in group...}

Q9. It is better that playing individually

Q10. It allows to get help from the group mates?

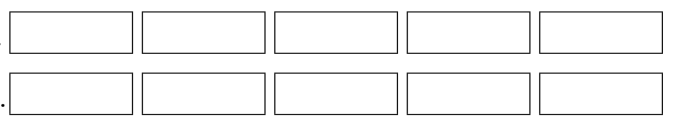

\section{Motivation}

Q11. I am interested to be in the podium
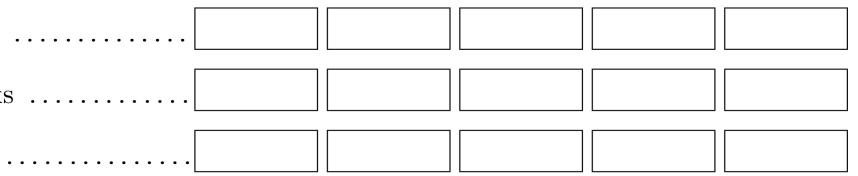

Q13. I am interested to pass the exams

For the future, Kahoot could be a good tool to evaluate in...

Q14. Theory

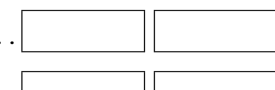

Q15. Classroom Practices

Q16. Computer Practices

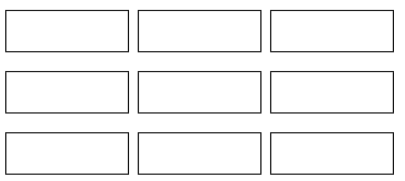

What qualification have you obtained in the first part of the course (theory and classroom practice)?

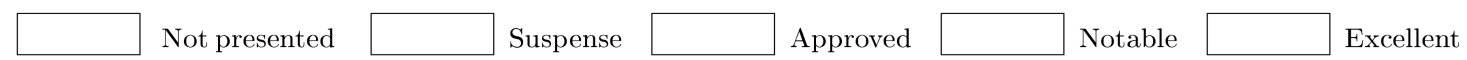

What qualification have you obtained in the first computer practices?

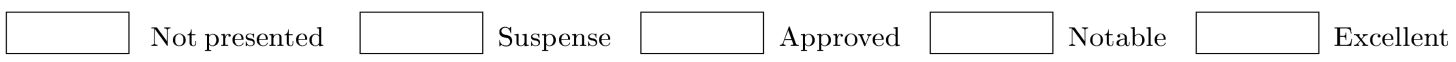

Fig. 6: Encuesta sobre la plataforma Kahoot. 


\section{Referencias bibliográficas}

Ankit, Deepak (2015). Quizz. URL: https://quizizz.com/.

Bryer, J. y K. Speerschneider (2016). "likert: Analysis and visualization likert items". En: $R$ package version 1.5 .

Coll Aliaga, P.D.C. y col. (2016). "Introducir Flip Education en nuestras aulas: Una experiencia en las prácticas de Matemáticas". En: XIV Jornadas de Redes de Investigación en Docencia Universitaria: investigación, innovación y enseñanza universitaria: enfoques pluridisciplinares. Instituto de Ciencias de la Educación, págs. 2200-2211.

Coll Aliaga, P.D.C. y col. (2017). "La metodología de clase inversa en la UPV: Una experiencia en el laboratorio de matemáticas". En: In-Red 2017. III Congreso Nacional de innovación educativa $y$ de docencia en red. Editorial Universitat Politècnica de València, págs. 417-428.

Cronbach, L. J. (1951). "Coefficient alpha and the internal structure of tests". En: psychometrika 16.3, págs. 297-334.

Cuzick, J. (1985). "A Wilcoxon-type test for trend". En: Statistics in medicine 4.4, págs. 543-547.

Göksün, D. O. y G. Gürsoy (2019). "Comparing success and engagement in gamified learning experiences via Kahoot and Quizizz". En: Computers \& Education 135, págs. 15-29.

Kapp, Karl M (2012). The gamification of learning and instruction: game-based methods and strategies for training and education. John Wiley \& Sons.

McKnight, P. E. y J. Najab (2010). "Mann-Whitney U Test". En: The Corsini encyclopedia of psychology, págs. 1-1.

Pérez Miras, S. D. (2017). "El uso de los dispositivos móviles en clase de Historia: experiencia de uso de Kahoot como herramienta evaluadora". En: DIM: Didáctica, Innovación y Multimedia 35, págs. 181-190.

Plickers (2019). plickers. URL: https://get.plickers.com/.

Revelle, W. R. (2017). psych: Procedures for personality and psychological research.

Rodríguez-Fernández, L. (2017). "Smartphones y aprendizaje: el uso de Kahoot en el aula universitaria". En: Edutec. Revista Electrónica de Tecnología Educativa 8 (1), págs. 181-190. DOI: https://www.doi.org/10.14198/MEDCOM2017.8.1.13.

Romero Claudio, C. y E. Álvarez Ramos (2020). "Kahoot! como recurso para (co)evaluar contenidos literarios en el aula de manera compartida. Una experiencia con estudiantes de $3 .^{\circ}$ de Secundaria". En: Revista Didáctica, Innovación y Multimedia 38, págs. 181-190. 
Segura, M. y col. (2019). "Evaluación interactiva del aprendizaje de Investigación Operativa basada en juegos mediante la plataforma Kahoot!" En: Congreso In-Red 2019 UPV, 11 y 12 de julio de 2019. Universitat Politècnica de València, págs. 1658-1671. DoI: http://dx.doi .org/10.4995/ INRED2019.2019.10381.

Sempere Ferre, F. (2019). "Kahoot como herramienta de autoevaluación en la universidad". En: Congreso In-Red 2018 UPV, 19 y 20 de julio de 2018. Universitat Politècnica de València, págs. 250-255. DOI: http://dx.doi.org/10.4995/INRED2018.2018.8730.

Soto García, I. S. de (2018). "Herramientas de gamificación para el aprendizaje de ciencias de la tierra". En: Edutec. Revista Electrónica de Tecnología Educativa 65, págs. 29-39. DOI: https: //doi.org/10.21556/edutec.2018.65.1143.

ThatQuiz (2019). ThatQuiz. URL: https://www.thatquiz.org/es/.

Zichermann, Gabe y Christopher Cunningham (2011). Gamification by design: Implementing game mechanics in web and mobile apps. .'Reilly Media, Inc.". 